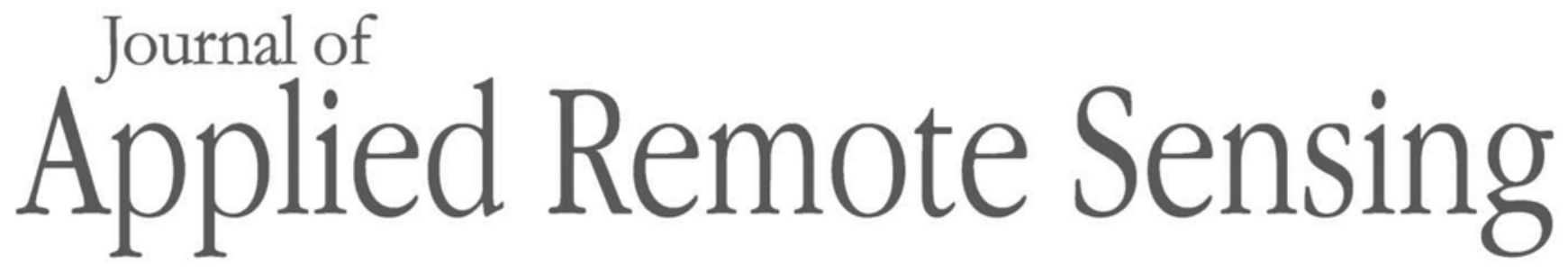

RemoteSensing.SPIEDigitalLibrary.org

\title{
Method for estimating rice plant height without ground surface detection using laser scanner measurement
}

\author{
Anh Thu Thi Phan \\ Kazuyoshi Takahashi \\ Atsushi Rikimaru \\ Yasuhiro Higuchi
}




\title{
Method for estimating rice plant height without ground surface detection using laser scanner measurement
}

\author{
Anh Thu Thi Phan, ${ }^{a}$ Kazuyoshi Takahashi, ${ }^{b, *}$ Atsushi Rikimaru, ${ }^{b}$ and \\ Yasuhiro Higuchic \\ ${ }^{a}$ Graduate School of Nagaoka University of Technology, 1603-1, Kami-Tomioka, \\ Nagaoka, Niigata 940-2188, Japan \\ ${ }^{b}$ Nagaoka University of Technology, 1603-1, Kami-Tomioka, Nagaoka, Niigata 940-2188, Japan \\ ${ }^{c}$ Niigata Agricultural Research Institute, 857 Nagakura, Nagaoka, Niigata 940-0826, Japan
}

\begin{abstract}
A method for estimating the height of rice plants, using three-dimensional laser range data from point clouds, is proposed and assessed. Rice plant height $(H)$ is estimated using a reference position at the top of the rice plant, avoiding the need to determine the ground position. Field experiments were performed with a SICK LMS 200 laser scanner in 2013 and 2014 on a test field with five different planting geometries. Percentile analysis identified the closest percentile to the top of the rice plant $\left(p^{t}=1\right)$, with vertical distances at the first percentile unaffected by planting geometry. The plant bottom position was identified using three different percentile ranks $\left(p^{\mathrm{b}}=95, p^{\mathrm{b}}=80\right.$, and $\left.p^{\mathrm{b}}=70\right)$. Relative vertical distances $(r D)$ were computed from the difference between the top and bottom positions of the rice plant. These correlated well with measured $H$, with slopes greater than 1.0. A greater number of stems in 2014 led to steeper slopes. Estimated $H$ was more accurate when plant bottom positions were closer to the ground surface, and the best results were obtained with $p^{\mathrm{b}}=95$ $\left(r^{2}>0.87 ; \mathrm{RMSE} \approx 4 \mathrm{~cm}\right)$. Overall, $H$ was typically $16.0 \mathrm{~cm}$ greater than $r D$ with $p^{\mathrm{b}}=95$. (C) The Authors. Published by SPIE under a Creative Commons Attribution 3.0 Unported License. Distribution or reproduction of this work in whole or in part requires full attribution of the original publication, including its DOI. [DOI: 10.1117/1.JRS.10.046018]
\end{abstract}

Keywords: rice plant height; relative vertical distance; laser scanner; percentile analysis.

Paper 16250 received Mar. 29, 2016; accepted for publication Nov. 7, 2016; published online Nov. 30, 2016.

\section{Introduction}

Rice is a major staple food in Asian countries, and the management of such a crop is vital for future food security. ${ }^{1}$ The demand for safe, affordable, and high-quality rice is increasing, and to satisfy these requirements, the rice production process needs to be carefully monitored and managed. Parameters such as plant height, the number of stems, the leaf area index, leaf color, fresh and dry weight, and crop growth rate are monitored over time in an attempt to control rice growth. ${ }^{2-6}$

In Japan, rice growth is typically indicated by rice plant height, the number of stems, and rice leaf color, all of which are measured periodically. ${ }^{7}$ The measurement of rice plant height, in particular, is considered a simple method for determining rice growth because this parameter, along with grain weight and spikelet per panicle, greatly influences the yield potential. ${ }^{8-10} \mathrm{~A}$ popular method for collecting crop growth information is to measure plant height and other growing parameters directly in the paddy field. However, it can be difficult to reach the plants, especially in a large field. The process of obtaining and recording measurements is monotonous, labor-intensive, and time-consuming, and the accuracy of the results depends on the number and spatial distribution of measured samples. In addition, if experiments are performed in many locations, with many plots, the results may contain errors from incorrect labeling or data recording. As a result, time- and labor-saving methods such as remote sensing techniques are considered a useful alternative and are widely utilized in agricultural production.

*Address all correspondence to: Kazuyoshi Takahashi, E-mail: ktakaha@nagaokaut.ac.jp 
Recently, laser scanners have proved to be an effective tool for precision agriculture, with their ability to collect large amounts of crop information in a relatively short time. ${ }^{11,12}$ In a previous study of plant height determination, Ehlert et al. ${ }^{13-15}$ proposed an approach of observing plant height in flat areas with a known ground level. The laser scanner is mounted on a tool carrier with an inclination angle $(\varphi)$ to observe the plant from above. The installation height of the sensor $\left(h_{s}\right)$ above the ground surface was presumed to be constant and was carefully determined and used as the reference value for calculating the height of the reflection point. The measured range, multiplied by the cosine of the scanning angle and the inclination angle of the sensor, was subtracted from $h_{s}$ to give the height of the reflection point above the ground surface. Zhang and $\mathrm{Grift}^{16}$ observed Miscanthus giganteus from a fixed point using a line laser scanner mounted sideways at a height of $50 \mathrm{~cm}$ above the ground surface. Additionally, the three-dimensional (3-D) structure of the crop could be determined by driving the sensor past a field edge. Following data collection, the top of the plant and the ground surface were extracted from 3-D laser point clouds, and crop height was determined as the distance between the two data points, accounting for the installation height of the laser scanner. Kaizu et al. ${ }^{17}$ presented a similar method of plant height estimation. In their study, a terrestrial laser scanner (TLS) was placed on a stable tripod outside the paddy field. Plant height was obtained from plant top altitude by subtracting the ground altitude, which was determined by uncovering the crop plant portion from laser point clouds. Furthermore, Hoffmeister et al. ${ }^{18}$ introduced the crop surface model (CSM), representing the top of the crop canopy, which was interpolated from laser point clouds collected by TLS. For this, the bare ground surface was obtained before cropping, and the map of plant height was generated by taking the difference between the CSM and the absolute height of the bare ground. Tilly et al. ${ }^{19-21}$ proposed an approach to estimate plant heights over time by establishing multitemporal CSMs from laser point clouds collected by a tripod-based TLS. In their study, crop growth was obtained at different time periods. Digital surface models (DSMs) were generated from observed laser point clouds. A digital elevation model (DEM), interpolated from the close to ground surface points in the sparser vegetation of the point clouds in the first observation, was used as a common reference surface for plant height calculation. Then, the CSMs were generated by subtracting the DEM from the respective DSM.

TLS has therefore been shown to be capable of estimating plant height. However, some problems remain with the application of this technique. In the studies mentioned, the laser scanner was always placed outside the paddy field, which means that scanner installation height is a significant factor in the measurement process. With lower installation heights, the laser beams are unable to reach the plants inside the paddy, whereas there was a huge difference in the scanning range when the laser scanner was installed at greater heights. In addition, observations of the ground surface or another reference surface close to the ground were required to compute plant height; however, their position cannot always be determined, especially in wetland paddies or among densely packed crops. Furthermore, the cost of crop-monitoring systems such as TLS, which includes initial outlay and running costs, contributes to the overall crop price, which affects consumers. Therefore, it is necessary to develop a system that is both effective at resolving these issues and incurs the lowest possible cost.

The present study proposes a new method for rice plant height estimation, which uses 3-D laser range data from point clouds and eliminates the need to detect the position of the ground surface. This presents the fundamental methodology for rice plant height monitoring with ground-based or small UAV-based laser scanner systems in the future. In this study, a new reference position is determined at the top of the rice plant; relative vertical distances are computed based on this reference position and are used to estimate rice plant height. In this way, the problem of unobtainable ground surface is addressed. To evaluate the proposed method, field observations were carried out in 2013 and 2014 with a scanner placed above the rice canopy.

\section{Experimental Site and Field Observations}

\subsection{Experimental Site}

The trial paddy is located in the Niigata Agricultural Research Institute in Niigata Prefecture, known as the rice capital of Japan. Under normal farming practices, paddy fields are plowed and 
Table 1 Planting density and geometry.

\begin{tabular}{lccc}
\hline \hline Plot & Planting density $\left(\right.$ plants $\left./ \mathrm{m}^{2}\right)$ & Planting geometry & $\begin{array}{r}\text { Row orientation relative } \\
\text { to scanning plane }\end{array}$ \\
\hline 1 & 21.2 & $30 \mathrm{~cm} \times 16 \mathrm{~cm}$ & $\perp$ \\
2 & 15.1 & $30 \mathrm{~cm} \times 22 \mathrm{~cm}$ & $\perp$ \\
3 & 11.2 & $30 \mathrm{~cm} \times 30 \mathrm{~cm}$ & - \\
4 & 15.1 & $22 \mathrm{~cm} \times 30 \mathrm{~cm}$ & $\|$ \\
5 & 21.2 & $16 \mathrm{~cm} \times 30 \mathrm{~cm}$ & $\|$ \\
\hline \hline
\end{tabular}

filled with water in April, and the rice-growing season starts in May and ends in late September. The rice was planted in two different paddy fields in two different years; otherwise, growing conditions, including rice variety, transplanting density, row direction, fertilizer treatments, and water supply were kept the same. The mean weather conditions were presumed to be unchanged between the two growing seasons. The test area was $2 \mathrm{~m} \times 15 \mathrm{~m}$ and was divided into five plots. Koshihikari, a popular rice variety in Japan, was planted with three different planting densities and two orientations (Table 1). The planting densities were classified as sparse, moderate, and dense, corresponding to $11.2,15.1$, and 21.2 plants $/ \mathrm{m}^{2}$, respectively, and one orientation was parallel to the movement of the rice-planting machine while the other was perpendicular to it [Fig. 1(b)]. Twenty day-old seedlings were transplanted in mid-May to the water-filled paddy field, which was treated with $3 \mathrm{~g} \mathrm{~N} / \mathrm{m}^{2}$ of ground fertilizer.

\subsection{Laser Scanner Instrument and Data Acquisition}

The instrument was set up to obtain a 3-D point cloud of the rice plant from above. The system included a line laser scanner (SICK LMS 200) and a slide rail along which the scanner could be moved. ${ }^{22}$ Both were hung from a rack at a height of $3 \mathrm{~m}$ from the ground surface, and the central scan angle of the laser scanner corresponded to the nadir. The scanning plane was set vertically downward and perpendicular to the row direction in plot 1 (Fig. 1). The field of view was $50 \mathrm{deg}$, with a scan angle from $-25 \mathrm{deg}$ to $25 \mathrm{deg}$; the angular resolution was $0.25 \mathrm{deg}$; and the laser beam wavelength was $905 \mathrm{~nm}$. The laser footprint diameter from the $3 \mathrm{~m}$ installation height ranged from 2.5 to $3 \mathrm{~cm}$, and the overlap in laser footprints within the scanning line was more than $60 \%$. Each scanning line had 200 scanning spots. To obtain the 3-D point cloud data, the laser scanner was moved along the slide rail for a distance of $\sim 1 \mathrm{~m}$ at a speed of $2 \mathrm{~cm} / \mathrm{s}$ under motorized power. This speed was chosen as the maximum velocity of the motor device. Figure 1 illustrates a schematic of the instrument on the rail in the test area. The setup described here,

(a)

(b)

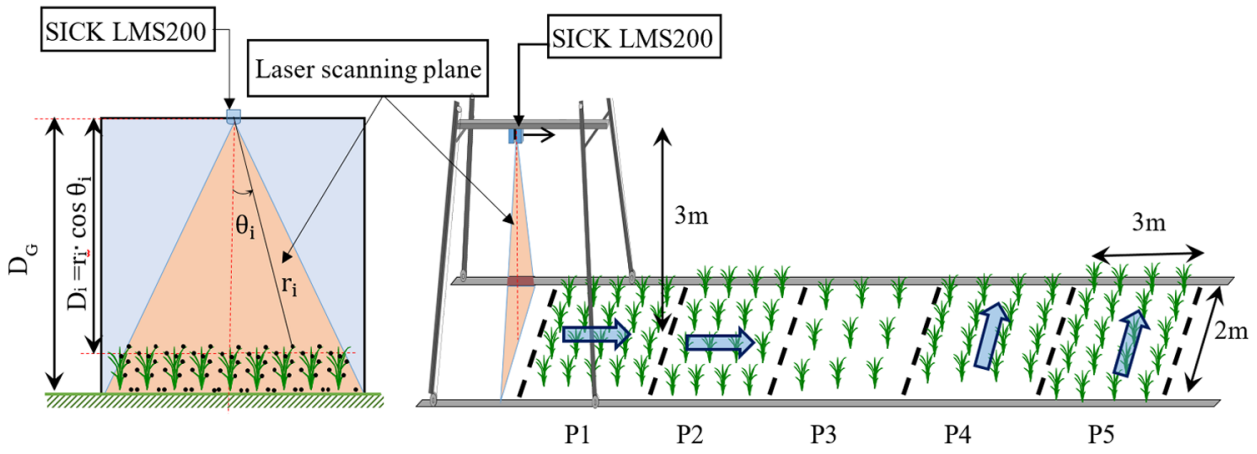

Fig. 1 Schematic of the field experiment setup for measuring the 3-D laser point cloud. (a) Laser scanning plane and (b) planting geometry of the five plots (P1 to P5). Arrows indicate row orientation. Row orientation is not considered in plot 3. 
in which the scanner is mounted upside down above the plant canopy, was previously used by Lumme et al. with a 3-D laser scanner for ear recognition and plant height determination. ${ }^{23}$

The measurements were carried out during the vegetative stage of growth, in which rice plant height rapidly increases. Sixteen observations were carried out in total between June and July of each year, and in only the first field observation of each year was the paddy field filled with water. Additional observations were made after the harvest in September, to obtain measurements from the bare soil, while a total station was also used to measure the elevation of the four corners of the target area in each plot. The data from both measurement methods were used to compute the ground position, thereby providing reference data with which the results of the proposed method could be validated. In addition to the acquisition of 3-D laser point clouds, rice plant height, the number of stems, and vegetation coverage were measured manually several times during the study period.

\section{Results}

\subsection{Manual Measurement Results}

According to the manual measurements made in this study, rice plant height $(H)$ increased linearly over time [Fig. 2(a)]. $H$ did not differ between the 2 years, probably because the same cultivar and fertilizer treatments were used. Moreover, rice plants in moderate density plots had higher $H$ than in other plots, with the maximum $H$ occurring in the moderate density plot with parallel row orientation. The largest detected difference between plots was $7 \mathrm{~cm}$ in 2013 and $4 \mathrm{~cm}$ in 2014. The standard deviation of average $H$ was $\sim 3 \mathrm{~cm}$ for the 2 years. Therefore, the effects of planting density and row orientation on $H$ are not thought to be significant in this study. Biswas et al. ${ }^{24}$ also stated that plant height did not differ significantly with differing planting geometry. However, the number of stems and vegetation coverage showed clear differences between the two growing seasons [Figs. 2(b) and 2(c)], with both parameters showing higher values in 2014. For example, 55 days after transplanting, the number of stems and vegetation coverage in 2014 were both 1.4 times larger than at the same time in 2013, despite rice plant height being the same in both years.

\subsection{Targeted Data Extraction}

The range data contained in the laser point clouds were converted to vertical distances $(D)$, which represent the distances between the scanning points and the installation height of the laser scanner [Eq. (1)]. Here, $D_{i}$ represents the vertical distance of the laser scanning points, $\theta_{i}$ is the inclination angle at the $i$ 'th position in the scanning line, and $r_{i}$ is the range from the sensor to the target at the $i$ 'th scan angle [Fig. 1(a)].

(a)

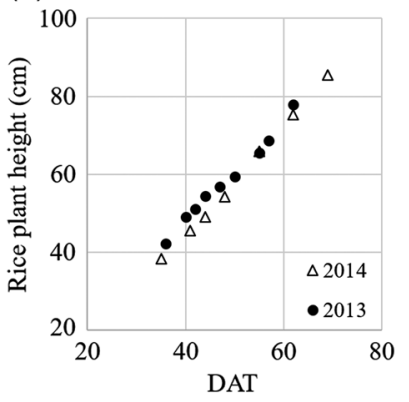

(b)

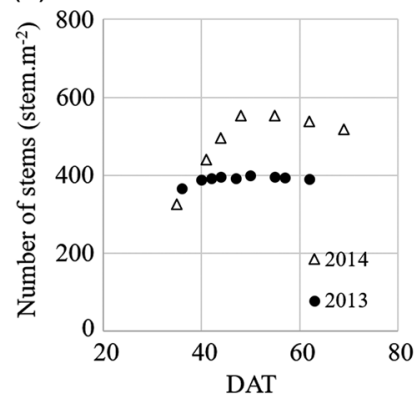

Days after transplanting (DAT) (c)

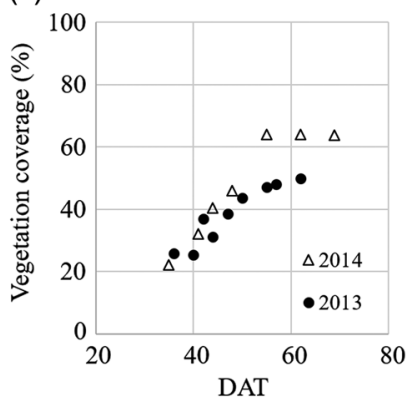
Fig. 2 Mean values of manually measured rice growth parameters. (a) Rice plant height, (b) number of stems, and (c) vegetation coverage. 
(a)

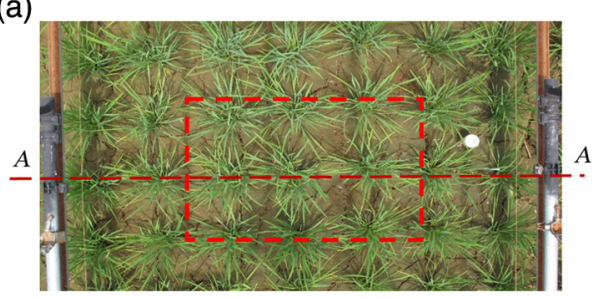

(c)

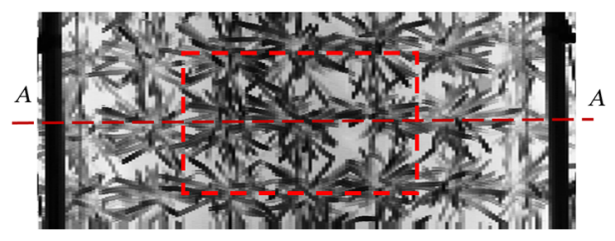

(b) 3.5

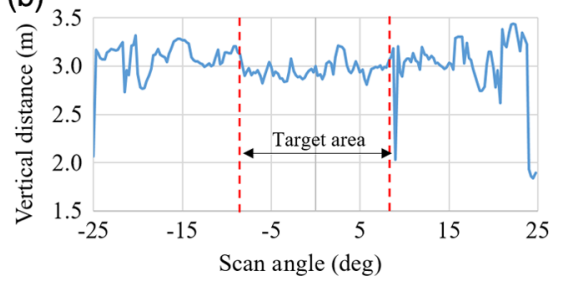

(d)

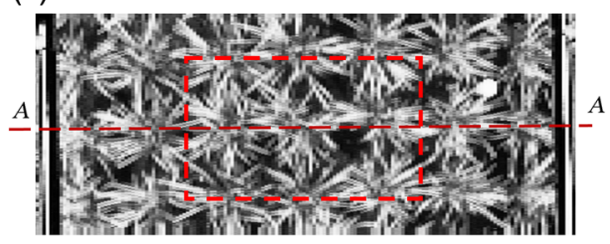

Fig. 3 Plot 3 target area in 2014. (a) Location of target area, (b) vertical distance relative to scan angle of scanline A-A in plot 3, (c) range image, and (d) intensity image.

$$
D_{i}=r_{i} \cos \theta_{i}
$$

To remove the effects of incident angle, small target areas $(90 \mathrm{~cm} \times 60 \mathrm{~cm})$ were selected inside each test plot within \pm 8 deg inclination angles [Fig. 3(a)]. The 3-D laser point clouds corresponding to the target areas were then extracted. Histograms plotting the percentage of laser pulses against vertical distance for plot 3 are shown in Fig. 4. In this, changes in the histogram shape indicate rice growth with time.

To determine the position of the ground surface, the average vertical distance to scanning points $\left(D_{G}\right)$ in bare ground data was used. Comparison of this with the total station measurement showed good agreement, as expected, and the difference of $1 \mathrm{~mm}$ in $D_{G}$ between the two methods is deemed insignificant. Thus, it was assumed that the collection of scanning points with a vertical distance approximately equal to $D_{G}$ represented a scanning of the ground surface. On this basis, the number of laser pulses reaching the ground surface was seen to decrease as rice growth progressed. However, paddy fields are typically filled with water, and in such situations it is difficult for the laser pulse to reach the ground surface because some laser wavelengths tend to be fully or partially absorbed by water. A simple test performed in 2013 showed that pulses from a SICK LMS 200 laser scanner could reach the ground surface beneath $4 \mathrm{~cm}$ of clear water, which is the standard water depth in the study area [Figs. 5(a) and 5(b)]. However, during the first field observation on June 18, 2014, there were many bubbles on the water surface and unclear water conditions prevailed [Fig. 5(c)]. Subsequently, it was found that with a

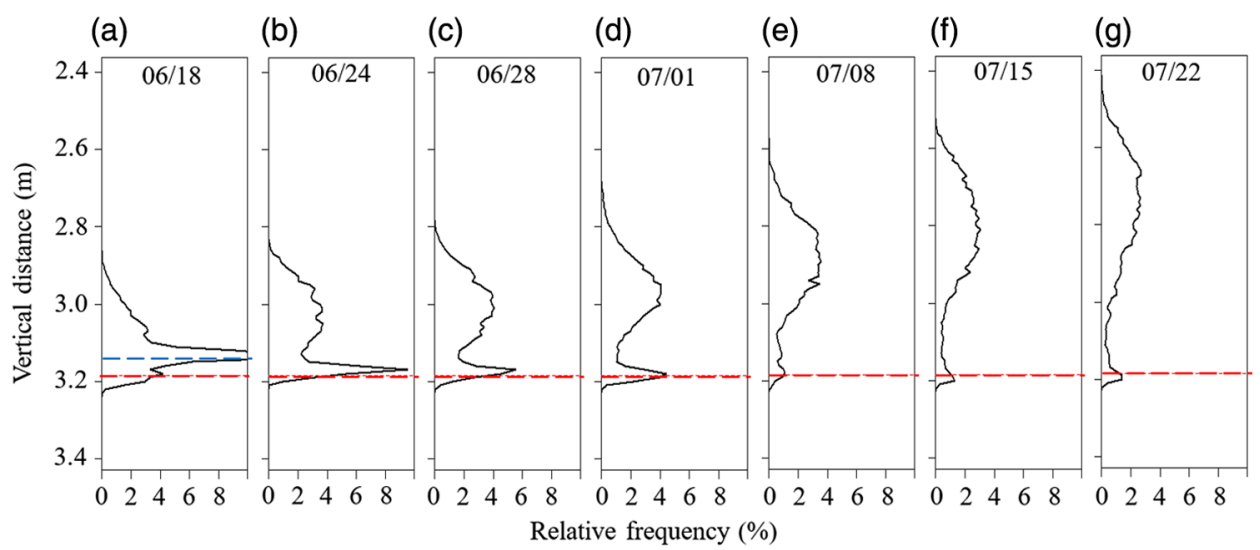

Fig. 4 Histograms of 3-D point cloud data from plot 3, collected in 2014. Red dashed lines show the ground position after harvesting the crop. Blue dashed line shows the water surface position in the first observation. In other field observations, the paddy was not filled with water. 
(a)

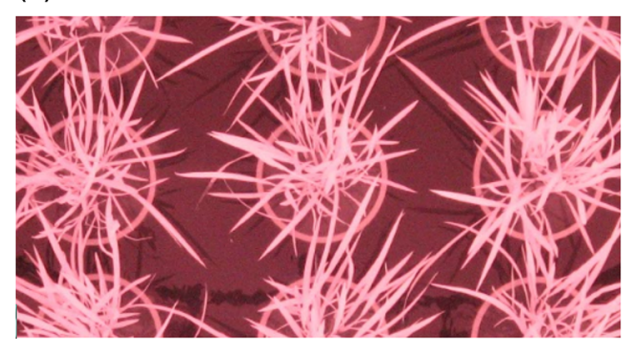

(c)

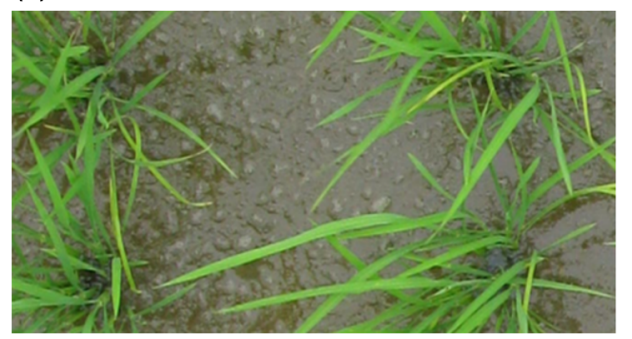

(b)

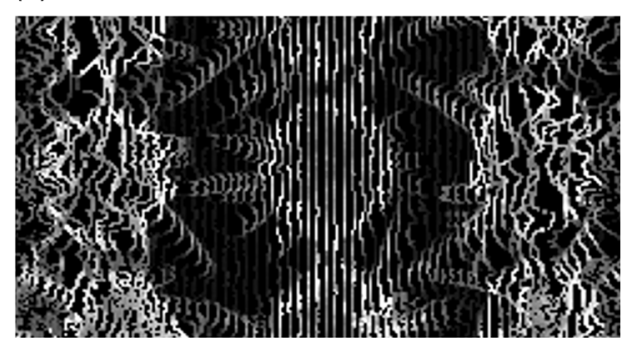

(d)

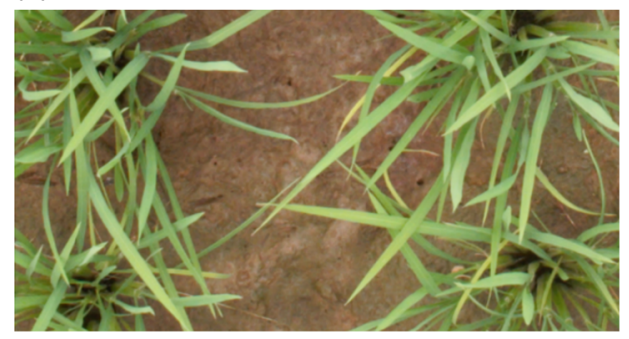

Fig. 5 The likelihood of SICK LMS 200 laser pulses reaching a ground surface covered by water. Images (a) and (b) are an infrared image and an intensity image collected by SICK LMS 200 on June 21, 2013, respectively. For this, potted rice plants were put into clear water, and the tops of the pots at a water depth of around $4 \mathrm{~cm}$ could be recognized from the intensity image. In contrast, (c) the paddy field was covered by turbid water with many bubbles on the water surface on June 18, 2014, and (d) the paddy field was not covered by water on June 24, 2014.

vegetation coverage of $20 \%$, more than $50 \%$ of laser pulses were absorbed by the water and did not reach the ground surface. Thus, the histogram peak in Fig. 4(a) indicates the position of the water surface and the red dashed line indicates the ground surface position directly beneath it. Fortunately, from the second observation in 2014 onward, the ground surface was not covered by water, so the laser pulse was able to reach the ground surface [Fig. 5(d)]. The histogram peaks closest to the horizontal axis indicate the position of the ground surface [Figs. 4(b)-4(g)]. It should be noted that the ground surface was typically uneven, with mounds around the rice plant trunks; this accounts for the small group of scanning points with smaller $D_{G}$ vertical distance values (Fig. 4).

\subsection{Estimation of Rice Plant Height}

Problems associated with the detection of the ground surface have already been discussed. It is possible to determine the ground surface shortly before transplanting or after harvesting the crop; however, in this study, the laser pulse easily reaches the rice canopy, and therefore we choose a new reference point at the top of the rice plant to determine $H$. The proposed method utilizes percentile analysis, which has been commonly used for analyzing plant heights obtained with a laser scanner. ${ }^{25,26}$ Therefore, a procedure using percentile ranks and vertical distance was performed, whereby the closest percentiles to the top $\left(p^{t}\right)$ and bottom $\left(p^{\mathrm{b}}\right)$ of the rice plant were determined. On the basis of this, the position of the top $\left(D^{\mathrm{t}}\right)$ and the bottom of the rice plant $\left(D^{\mathrm{b}}\right)$ were then located (Fig. 6).

First, the top of the rice plant $\left(D^{\mathrm{t}}\right)$ was identified. During rice crop monitoring, numerous factors such as wind, insects, or small airborne particles affect laser scanning data. Therefore, we assume here that $<1 \%$ of laser scanning points were affected by these factors. A search procedure was performed to identify $D^{\mathrm{t}}$. The vertical distances at difference percentile ranks between 0 and 100 were computed from laser point clouds, with an equal interval of 1.0. The first percentile closest to the top of the rice plant was confirmed, and the vertical distance corresponding to the first percentile $\left(D^{t}\right)$ was considered to be located at the top of the rice plant. This did not differ significantly between the various plots, and the standard deviation in $D^{\mathrm{t}}$ was $\sim 4 \mathrm{~cm}$ over the 2 
Thi Phan et al.: Method for estimating rice plant height without ground surface detection...

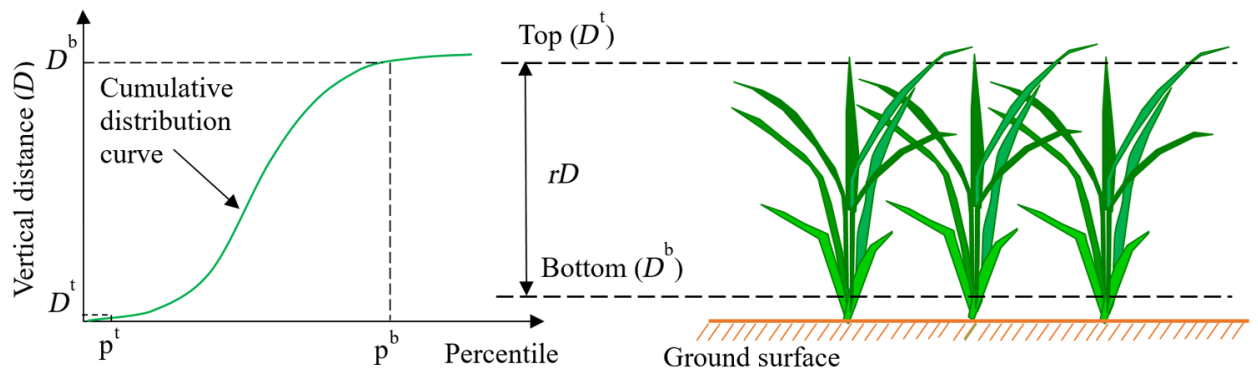

Fig. 6 Determination of the top and bottom location, where $p^{t}$ and $p^{b}$ are the closest percentiles to the top and bottom of the rice plant, respectively; $D^{\mathrm{t}}$ and $D^{\mathrm{b}}$ are the vertical distances from the scanning point to the installation height of the laser scanner at $p^{t}$ and $p^{\mathrm{b}}$.

years. Thus, it appears that the effect of planting geometry on vertical distance at the first percentile was not considerable in this study.

In the next step, the position of the bottom of the plant $\left(D^{\mathrm{b}}\right)$ was identified. Three percentile ranks (95th, 80th, and 70th) were chosen as the closest percentiles to the bottom of the rice plant. Figure 7 shows that the vertical distance at the 95th percentile rank is the most similar to $D_{G}$, whereas the vertical distance at the 70th percentile rank shows the greatest difference. For the

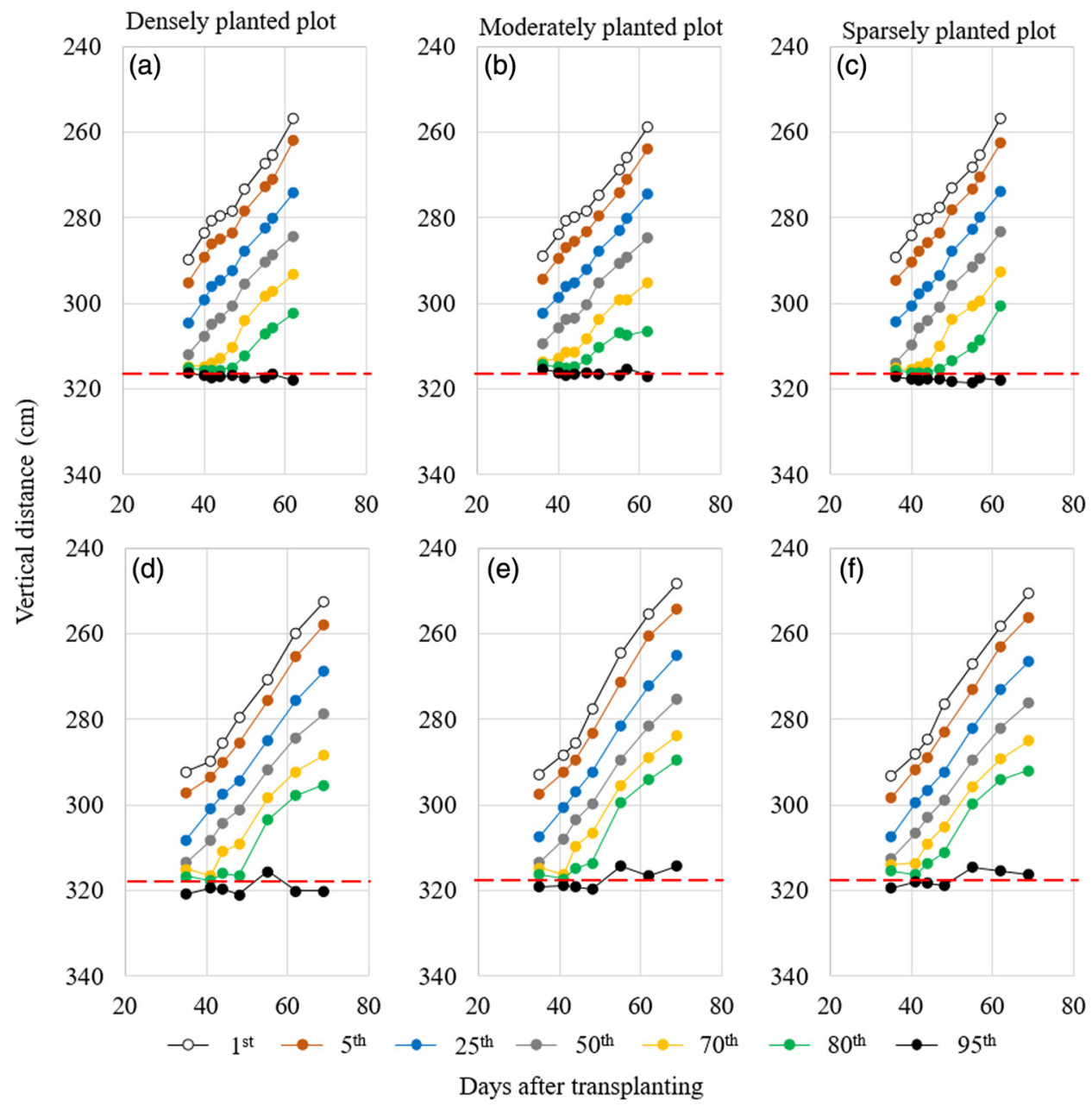

Fig. 7 Vertical distance at various percentile ranks. (a-c) Results from 2013 and (d-f) from 2014. Columns show results from ( $a$ and $d$ ) densely planted plots, $(b$ and $e$ ) moderate density plots, and ( $c$ and $f$ ) sparse plots. Red dashed lines show the position of the ground surface after harvesting the crop. 
80th percentile rank, the vertical distance approximates $D_{G}$ in the first 40 or 50 days but diverges from $D_{G}$ as time progresses (Fig. 7).

Finally, relative vertical distances $(r D)$ were calculated, as the distance between the top and bottom of the rice plant (Fig. 6). Figure 8 illustrates a correlation in all cases when $r D$ is plotted against $H$. The regression lines show a high coefficient of determination $\left(r^{2}\right)$; however, these results depend on the input data and the chosen bottom position. The strongest correlations were identified with $p^{\mathrm{b}}=95$, with $r^{2}>0.86$ [Figs. 8(c), 8(f), and 8(i)], and the value of $r^{2}$ decreases with smaller $p^{\mathrm{b}}$. In 2013, data from the last four observations from plot 4 differ significantly from the general trend, leading to the low $r^{2}$ of 0.48 when $p^{\mathrm{b}}=70$ [Fig. 8(a)].

It should be noted that narrow rice leaf blades, which can be smaller in width than the laser footprint size, lead to difficulties in detecting both the rice leaf tip and the individual rice leaves with the laser scanner. Therefore, $r D$ was always smaller than $H$, such that all regression lines have a slope greater than 1.0. The slopes differs between the two years; as cultivation conditions were kept the same, this could be attributed to a difference in the number of stems. A larger number of stems appears to correspond to steeper slopes, as in 2014 all regression line slopes are steeper than those of 2013. Hence, the number of stems should be considered carefully in future studies.

To evaluate the new method detailed here, estimated rice plant heights were compared with measured $H$. The smallest root mean square error $(\mathrm{RMSE} \approx 4 \mathrm{~cm})$ was achieved when the calculated plant bottom position was closest to the ground surface, when $p^{\mathrm{b}}=95$. For the 80 th and
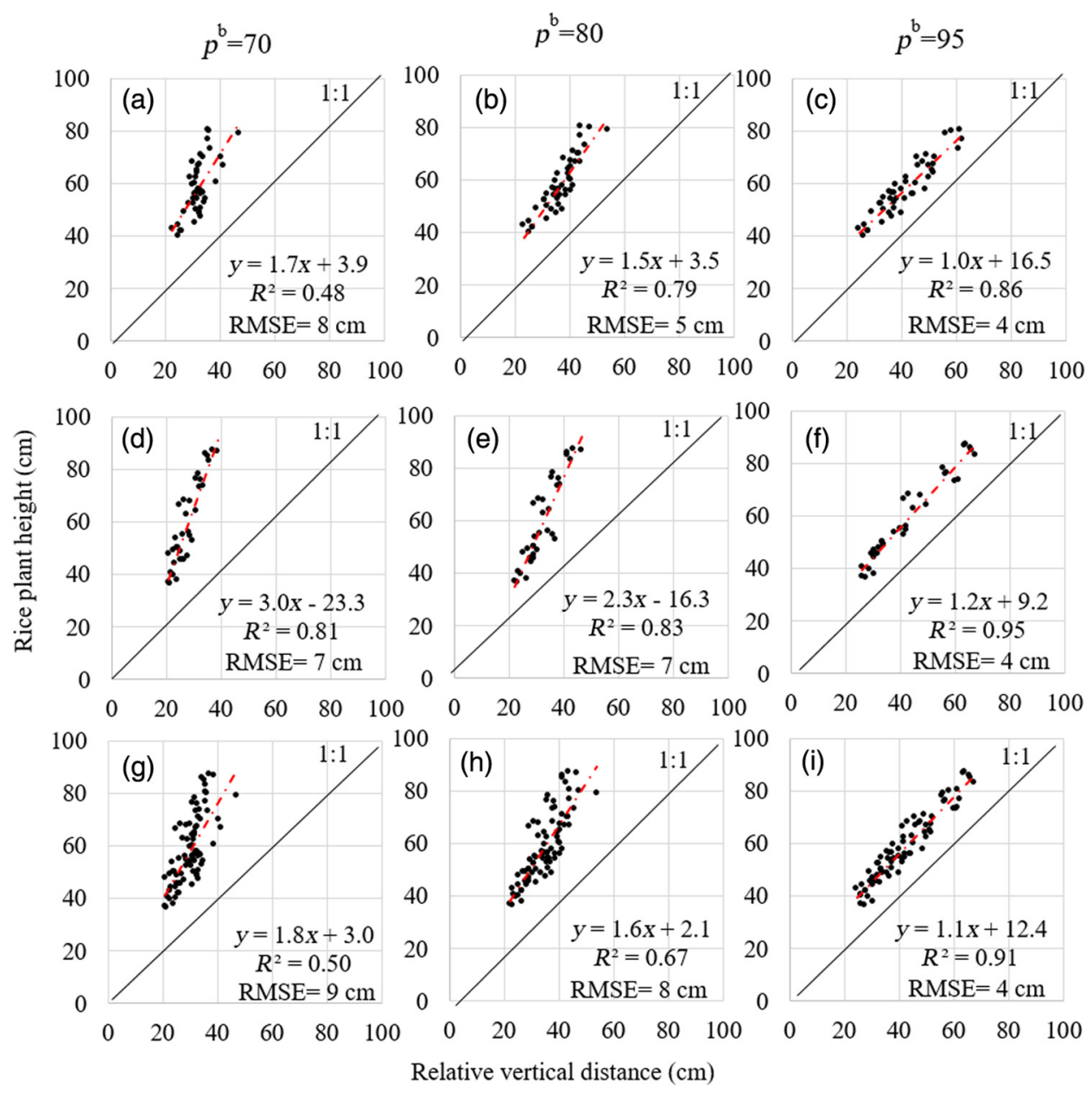

Fig. 8 Plots of $r D$ against measured rice plant height. The rows show the results from $(a-c) 2013$, (d-f) 2014, and $(\mathrm{g}-\mathrm{i})$ a combination of the 2 years. The columns show the results with the reference position computed at $(\mathrm{a}, \mathrm{d}, \mathrm{g})$ 70th, $(\mathrm{b}, \mathrm{e}, \mathrm{h})$ 80th, and $(\mathrm{c}, \mathrm{f}, \mathrm{i})$ 95th percentile ranks. Red dashdotted lines show the regression lines. 
70th percentiles, the RMSE was 8 to $9 \mathrm{~cm}$ when data from both years were combined. All other positions of the plant bottom with $p^{\mathrm{b}}<70$ determined above the ground surface gave acceptable results (RMSE $\leq 10 \mathrm{~cm}$ ), although it should be noted that plant bottom positions were limited to above the 50th percentile. These results illustrate the capability of this new method to estimate plant height from 3-D laser point cloud data. Furthermore, we identified a constant bias between $H$ and $r D$ with $p^{\mathrm{b}}=95$ [Figs. 8(c), 8(f), and $8(\mathrm{~g})$ ]. By forcing the slopes of the regression lines of these cases to $1.0, H$ was found to be $16.0 \mathrm{~cm}$ greater than $r D$.

\section{Discussion}

To solve the problem of unobservable ground surface, especially in densely planted or wet-land paddies, the position of the top of rice plant is determined and used as a new reference position to estimate $H$. This study found that planting density and row direction did not significantly influence both $H$ and the value of $D^{\mathrm{t}}$ at $p^{\mathrm{t}}=1$. The position of the top of rice plant could be identified at the first percentile rank and used as reference position for estimating rice plant height. $r D$ was computed at three chosen plant bottom positions $\left(p^{\mathrm{b}}=70, p^{\mathrm{b}}=80\right.$, and $p^{\mathrm{b}}=95$ ), and consistent regression lines (all with slopes greater than 1.0) were determined, with a greater number of stems contributing to steeper slopes. Without considering differences in the number of stems, RMSE was consistently less than $9 \mathrm{~cm}$. The best results in this study were obtained with $p^{\mathrm{b}}=95\left(r^{2}>0.86 ; \mathrm{RMSE} \approx 4 \mathrm{~cm}\right)$. For comparison, $r^{2}$ corresponding to plant growth of 0.86 and 0.91 were also introduced by Tilly et al. ${ }^{17,19}$ in their studies on plant growth. Bendig et al. ${ }^{6}$ derived CSMs from UAV-based red green blue imaging and reported an $r^{2}$ of 0.92 on a summer barley crop. Additionally, plant height estimation errors reported by Zhang and Grift, ${ }^{16}$ Kaizu et al., ${ }^{17}$ and Tilly et al. ${ }^{21}$ were 14 , nearly 10 , and $5 \mathrm{~cm}$, respectively. The RMSE of $4 \mathrm{~cm}$ in this study does not differ significantly from the $3 \mathrm{~cm}$ standard deviation of the manually measured $H$, and corresponds to $10 \%$ and $5 \%$ of the measured rice plant heights in the first and last observations, respectively. Therefore, the results obtained using the proposed method for estimating rice plant height with RMSE are more accurate than those in the aforementioned studies. Moreover, a constant bias was identified between $H$ and $r D$ with $p^{\mathrm{b}}=95$; $r D$ was $16 \mathrm{~cm}$ lower than $H$ on average and the RMSE was $4 \mathrm{~cm}$. Thus, this method is suitable for obtaining rice plant heights greater than $20 \mathrm{~cm}$. Using $r D, H$ can be estimated in situations where the ground surface is unobservable or has some notable relief. In this manner, observation cost is reduced through decreased time and labor requirements for measuring the ground surface or extracting it from observation data. ${ }^{16,17}$

In addition to the suitable results, a number of limitations must be considered carefully in future studies. In particular, the accuracy of the results is largely dependent on the laser footprint size of the specific laser scanner used. When the laser footprint is large, it is difficult to capture individual rice leaves, lowering the accuracy of the results. Moreover, in this study, the small target areas selected were observed in nadir without considering incident angle effect; therefore, the results might not be applicable to an entire paddy field. Future studies should consider the influences of incident angle and carefully consider the number of stems, which was not considered in this study.

\section{Conclusion}

This study raised and solved an important problem regarding rice plant height $(H)$ estimation without ground surface detection using laser scanner measurement. In the proposed method, a new reference position is determined at the top of the rice plant, and relative vertical distances $(r D)$ based on the new reference position are used to estimate $H$. The results showed that $r D$ has a high correlation with $H$, and consistent regression lines with greater than 1.0 slopes were determined. With increased distance between the plant bottom position and the ground surface, the effect of the number of stems on the regression slopes also increased. Additionally, by employing $r D$, the positions of ground surface need not be considered. When the identified bottom position of the plant was closer to the ground surface, the achieved RMSE of $4 \mathrm{~cm}$ was $1 \mathrm{~cm}$ greater than standard deviation of manually measured $H$. With RMSEs ranging 
from 4 to $10 \mathrm{~cm}$ in nadir observation, the proposed method can be possibly applied to large-scale observations of rice crop using ground-based or UAV-based LiDAR with no extra cost for identifying ground surface position. In future studies, the effect of the number of stems on the correlation between estimated and measured $H$ should be considered, in addition to careful investigations into the effects of incident angle. In this way, the proposed methodology can be developed and accurately applied to larger target areas.

\section{Acknowledgments}

This study was partially supported by JSPS KAKENHI Grant Nos. 23580361 and 26450362.

\section{References}

1. International Rice Research Institute (IRRI), "The importance of rice," (29 March 2015) http://www.knowledgebank.irri.org/ericeproduction/bodydefault.htm\#Importance_of_Rice. htm

2. A. Dobermann and M. F. Pampolino, "Indirect leaf area index measurement as a tool for characterizing rice growth at the field scale," Commun. Soil Sci. Plant Anal. 26(9-10), 1507-1523 (1995).

3. T. Takai et al., "Rice yield potential is closely related to crop growth rate during late reproductive period," Field Crops Res. 96, 328-335 (2006).

4. M. Hasanuzzaman et al., "Plant growth pattern, tiller dynamics and dry matter accumulation of wetland rice (Oryza sativa L.) as influenced by application of different manures," Nat. Sci. 8(4), 1-10 (2010).

5. B. Singh et al., "Fixed-time adjustable dose site-specific fertilizer nitrogen management in transplanted irrigated rice (Oryza sativa L.) in South Asia," Field Crops Res. 126, 63-69 (2012).

6. J. Bendig et al., "Estimating biomass of barley using crop surface models (CSMs) derived from UAV-based RGB imaging," Remote Sens. 6, 10395-10412 (2014).

7. Y. Yamamoto, S. Kawaguchi, and W. Takahashi, "Application of developmental model for analysis of growth and development of rice transplanted in different plant numbers per hill," Jpn. J. Crop Sci. 63(2), 208-214 (1994).

8. J. B. Thomas, G. B. Schaalje, and M. N. Grant, "Height, competition and yield potential in winter wheat," Euphytica 74, 9-17 (1994).

9. M. G. S. Fernandez et al., "From dwarves to giants: plant height manipulation for biomass yield," Trends Plant Sci. 14(8), 454-461 (2009).

10. X. F. Bai et al., "Quantitative trait loci for rice yield-related traits using recombinant inbred lines derived from two diverse cultivars," Genetics 90(2), 209-215 (2011).

11. F. Hosoi and K. Omasa, "Estimating vertical plant area density profile and growth parameters of a wheat canopy at different growth stages using three-dimensional portable lidar imaging," ISPRS J. Photogramm. Remote Sens. 64, 151-158 (2009).

12. F. Hosoi and K. Omasa, "Estimation of vertical plant area density profiles in a rice canopy at different growth stages by high-resolution portable scanning LIDAR with a lightweight mirror," ISPRS J. Photogramm. Remote Sens. 74, 11-19 (2012).

13. D. Ehlert, H. J. Horn, and R. Adamek, "Measuring crop biomass density by laser triangulation," Comput. Electron. Agr. 61(2), 117-125 (2008).

14. D. Ehlert, H. J. Horn, and R. Adamek, "Suitability of a laser rangefinder to characterize winter wheat," Precis. Agr. 11, 650-663 (2010).

15. D. Ehlert and M. Heisig, "Sources of angle-dependent errors in terrestrial laser scannerbased crop stand measurement," Comput. Electron. Agr. 93, 10-16 (2013).

16. L. Zhang and T. E. Grift, "A LIDAR-based crop height measurement system for Miscanthus giganteus," Comput. Electron. Agr. 85, 70-76 (2012).

17. Y. Kaizu, J. M. Choi, and T. H. Kang, "Grass height and yield estimation using a threedimensional laser scanner," Environ. Control Biol. 50(1), 41-51 (2012).

18. D. Hoffmeister et al., "High resolution crop surface models (CSM) and crop volume models (CVM) on field level by terrestrial laser scanning," Proc. SPIE 7840, 78400E (2010). 
Thi Phan et al.: Method for estimating rice plant height without ground surface detection...

19. N. Tilly et al., "Evaluation of terrestrial laser scanning for rice growth monitoring," Int. Arch. Photogramm. Remote Sens. Spatial Inf. Sci. XXXIX-B7, 351-356 (2012).

20. N. Tilly et al., "Precise plant height monitoring and biomass estimation with terrestrial laser scanning in paddy rice," ISPRS Ann. Photogramm. Remote Sens. Spatial Inf. Sci. II-5/W2, 295-300 (2013).

21. N. Tilly et al., "Multitemporal crop surface models: accurate plant height measurement and biomass estimation with terrestrial laser scanning in paddy rice," Appl. Remote Sens. 8, 1-22 (2014).

22. SICK AG, "LMS200/211/221/291 Laser Measurement Systems," http://sicktoolbox. sourceforge.net/docs/sick-lms-technical-description.pdf (20 March 2014).

23. J. Lumme et al., "Terrestrial laser scanning of agricultural crops," Int. Arch. Photogramm. Remote Sens. Spatial Inf. Sci. 5, 563-566 (2008).

24. M. Biswas, A. H. M. M. Rahman, and F. Ahmed, "Effect of variety and planting geometry on the growth and yield of hybrid maize," J. Agr. Environ. Sci. 3(2), 27-32 (2014).

25. D. Riano et al., "Modeling airborne laser scanning data for the spatial generation of critical forest parameters in fire behavior modeling," Remote Sens. Environ. 86(2), 177-186 (2003).

26. M. Hämmerle and B. Höfle, "Effects of reduced terrestrial LiDAR point density on highresolution grain crop surface models in precision agriculture," Sensors 14, 24212-24230 (2014).

Biographies for the authors are not available. 